\title{
Controlled Flooding with Passive Anti-Flooding for Urgent Messages in Body Area Sensor Networks
}

\author{
Jinze Yang, Yan Sun, Jesus Requena Carrion \\ Electronic Engineering and Computer Science School \\ Queen Mary University of London \\ London \\ \{jinze.yang, yan.sun, j.requena\}@qmul.ac.uk
}

\begin{abstract}
This paper proposes a novel routing scheme, named Controlled Flooding with Passive Anti-Flooding (CFPAF), aiming at handling Urgent Messages (UMs) in Body Area Sensor Network (BASN). By contrast to most of existing routing schemes, which employ common flooding methods for forwarding UMs towards the destination, CFPAF combines a Controlled Flooding approach with Passive Anti-Flooding capability for forwarding UMs while reducing the transmission cost. In addition, Advance Gossip Mechanism is adopted in CFPAF to further reduce the UM flooding storm. Simulation results show that compared to the classic Multi-Path Forwarding method, CFPAF dramatically reduces the number of forwarded packages without significantly affecting the average end-to-end delay.
\end{abstract}

Keywords—urgent message handle, BASN, energy saving

\section{INTRODUCTION}

Wireless sensor networks (WSNs) have been a popular research topic in recent years. One of the most promising application areas of WSN is healthcare and fitness. By placing devices which are equipped with sensing, processing and communication capabilities, near, on or inside the body, Body Area Sensor Networks (BASN) can enable healthcare and fitness applications to monitor and process physiological data. Due to the electromagnetic properties of the human body and the nature of the applications, BASN faces specific challenges compare to conventional WSN [1,2]. Firstly, the wireless channel close to or inside human body presents a high loss rate [3]. Secondly, energy is limited because of small battery size and the difficulty of re-charging. Finally, event triggered data leads to uncertain traffic, which is consequently hard to predict [2].

Urgent messages (UMs) constitute one type of eventtriggered messages that usually carry critical information in healthcare applications. BASNs can generate UMs carrying abnormal physiological data, which can be used as an alarm for medical professionals. For example, life-threatening cardiac episodes in patients wearing BASN can be reported by using UM. However, high end-to-end delay and packet loss rate can degrade the effectiveness of UMs. Generally, there exist two main strategies to handle UMs. One solution is maintaining a dedicate route for UM, which guarantees low end-to-end delay for UM delivery. However, maintaining dedicate route can be costly due to the low frequency of UM. Another solution, which establishes a route after urgent events occur, might save more resources, but leads to higher end-to-end delays. The challenge of effectively handling UM is therefore to reduce the end-to-end delay while minimizing the usage of resources. Previous studies have tackled the challenges of handling UM in general WSN. In [4], the authors introduced a priority mechanisms for IEEE 802.3 to reduce the end-to-end delay for UM delivery. In [5], a protocol was proposed to choose a route based on end-to-end delay requirement. However, neither [4] nor [5] addresses the effects on the energy consumption. In [6], a cross-layer protocol with priority was developed to balance the end-to-end delay and energy consumption. The protocol turns off the radio module periodically to save energy. However, when UMs are generated, all nodes remain active to guarantee the UM delivery. In [7], UMs generated by the WSN were forwarded by a special directional flooding mechanism. It is worth noting that although authors in [6] and [7] aimed at saving energy during normal data transmission while guaranteeing the delivery of UM, other studies have focused on saving the energy required specially for delivering UM. Authors in [8] proposed a mechanism focusing on route repair. This mechanism either implements sequential repair or uses a backup route to avoid reparation. However, it does not take the random nature of the UM generation into consideration.

This paper proposes a Controlled Flooding with Passive Anti-Flooding (CFPAF) protocol to handle UMs in BASN. The CFPAF protocol focuses on route repair mechanism to reduce the cost of UM delivery and balance end-to-end delay. This paper is organized as follows. Section II describes the existing literature in details about handling UM in WSN. Section III introduces the CFPAF protocol for BASN. Section IV discusses the simulation scenarios and the results. Section V draws the conclusions of this paper.

\section{Protocol BACKGROUND}

As mentioned in previous section, UMs requires low end-toend delay while BASN desires minimum cost of delivery. Maintaining a route for UM is costly in BASN while it suffers long delay to search for a route when an UM is already generated. Many researchers have proposed solutions to this problem, which can be classified into two main categories: with and without pre-established route. 


\section{A. Solutions without pre-established route}

Without a pre-established route, one way to shorten the endto-end delay is flooding the UMs in the whole network. As a most reliable way to forward information, flooding has been introduced in many aspects of Mobile Ad hoc Network (MANET), for example, the broadcast of Route Requests (RREQs) to find a suitable route to destination. The main disadvantage of flooding is that all nodes will occupy the wireless channel at the same time, which leads to heavy interference. The issues is known as flooding storm.

One classic way to reduce the size of flooding storm is called gossip. Gossip consists of a series of mechanisms for reducing the number of broadcasted messages. If the radio range is potentially high overlapping, it is possible that the whole network area can be covered if only some of the nodes broadcast the message. Then the problem sits in which nodes should perform broadcasting. Authors in [9] introduced a few gossip schemes for MANET. In [10], several schemes proposed target in Vehicular Ad hoc wireless Networks (VANET). Schemes mentioned in [9] and [10], which choose a set of nodes for broadcasting, are classified into counter based probabilistic scheme and distance based probabilistic scheme. Therefore, the probability of rebroadcasting varies due to different formulas of different scheme. Besides reducing the number of broadcast nodes in whole network, limit the broadcasting range within partial network coverage might further reduce the number of broadcasted messages.

Some researchers introduced other mechanisms to replace flooding completely. As introduced in [11], the query control solution is a mechanism to reduce the number of quires in Zone Routing Protocol (ZRP). The query is adopted to replace the RREQ and is multi-casted to a node's two hop away neighbours when searching for a route. The duplicated queries can be discovered and discarded by forwarding nodes. Therefore, the total number of queries are reduced. Query control mechanism is complicated and it introduces extra computing cost which unfortunately is also one of the limited resources in BASN.

The reason why RREQs need to cover the whole network is that the source node cannot know where the destination is. If the direction of destination node is available to all the nodes in the network, the number of necessary RREQs can be reduced. In [12], a proactive protocol was introduced. When network is initializing, the sink node or destination will flood the control messages over the whole network. Nodes which receive the control messages will record how many hops they are away from the sink node, indicating by the hop distance to sink node. When a source node need to send a UM, it will choose two neighbours as two next hops and send the UM to two next hops twice. The duplicated messages are used to avoid packet loss. The assumption in [12] is that the data loss due to node loss is rare, and [12] does not take nodes' mobility in to consideration. If the nodes are moving frequently, this protocol will need to update the hop distance frequently which might consume a great deal of resource.

\section{B. Solutions with pre-established route}

When a pre-established route exists, the common solution to shorten the end-to-end delay is to have a backup route. If the original route is broken, the backup route will be used instantly. In [13], when constructing a route, the protocol select a few backup nodes which are one hop away from the main route. When route break happens, the designed mechanism will repair the route with backup nodes. These backup nodes will reduce the end-to-end delay by reducing the route repair time as well as avoiding flooding storm.

The protocol proposed in [14] is called Multi-Path Forwarding (MPF). MPF establishes co-existing routes from source node to destination node. The UMs will go through all co-existing routes. Duplicated messages reduce the risk of UM packet loss. When a route is broken, other routes might still be available. Therefore, UM can travel through other valid routes without waiting for route reconstruction.

The main disadvantage of the two protocols with back up routes proposed in [13] and [14] is: periodically sending maintaining messages will shorten the lifetime of the whole network. Due to low UM rate, routes for UM are not required in most of the network working time.

The main contributions in this work are listed below:

- A new control packet Route Exist (REXT) is designed to terminate the flooding storm;

- An additional five route states are introduced as a state machine in CFPAF mechanisms;

- The Passive Anti-Flooding with Advanced Gossip Mechanism (PAF-AGM) mechanism is proposed with the function of terminating flooding storm and saving resources.

\section{Protocol Design}

The principle of CFPAF is that when the route break happens, the UM follows economic flooding scheme while repairing the route to the final destination. Two methods are adopted in CFPAF to reduce the cost of flooding: Controlled Flooding (CF) and PAF-AGM. The CF mechanism aims at reducing end-to-end delay during route reconstruction, while PAF-AGM further reduces the cost of flooding as well as saves energy consumption for the whole network.

\section{A. Newly designed control packet types in CFPAF}

The Route Exist (REXT) message is specially designed in CFPAF with the function of updating the route states during UM flooding. REXT is broadcasted to two hops away so the TimeTo-Live (TTL) is set as 2, and all the network nodes that receive the REXT message will update their route table accordingly and reduce TTL by one. This detailed process is described in Section III-B.

\section{B. Newly Designed Route states}

In CFPAF, five new route states in the route table are designed. The meaning of each state is listed in Table I. To make it simple, in the following parts of the paper, "the node have a 
route table which is in RT_OK state" will be rephrased as "the node in RT_OK state".

TABLE I ROUTE STATES

\begin{tabular}{|l|l|}
\hline RT_OK & $\begin{array}{l}\text { The node has a route from source to destination and the } \\
\text { route has a valid next hop. }\end{array}$ \\
\hline RT_LOSS & $\begin{array}{l}\text { The node had route once but the next hop is lost. Route } \\
\text { table is still kept in memory. }\end{array}$ \\
\hline PAF_S & $\begin{array}{l}\text { The node has overheard Route Reply (RRPY) or } \\
\text { receives REXT. It performs Passive Anti-Flooding } \\
\text { (PAF) function. }\end{array}$ \\
\hline RT_REP & $\begin{array}{l}\text { The node received broadcasted UM and has sent it. It is } \\
\text { a candidate of the break route. }\end{array}$ \\
\hline IDLE & $\begin{array}{l}\text { The node does not receive any UM and does not have } \\
\text { UM route table. }\end{array}$ \\
\hline
\end{tabular}

An example about how the new route state works is given here. When a route for transmitting UM is established, the nodes on the route are in RT_OK state. If the next hop of one node is lost, the node will enter the RT_LOSS state instead of sending a Route Error (RERR) to source. Nodes in RT_OK and RT_LOSS state have long route expire time because these nodes can indicate the rough direction of the destination.

\section{Controlled Flooding (CF)}

When a node receives an $\mathrm{UM}$, and the route state is RT_OK, the UM will be simply forwarded to next hop. However, if the route state is RT LOSS, the Controlled Flooding (CF) is triggered and the UM will be broadcasted. Note that the UM is broadcast at the end node of the break route rather than the source node. When nodes who are currently not on any routes receive the UM, they will create their route table with RT_LOSS state set and prepare to broadcast the UM after a random backoff time $r t$. The back-off time $r t$ is uniformly distributed in the interval $\left[0,2 \times b a c k \_o f f\right]$. If nodes are still in RT_LOSS state after the back-off time $r t$, the UM will be broadcasted following the Advanced Gossip Mechanism (AGM) scheme described in Section III-E. Another function of $\mathrm{CF}$ is to perform route reparation. Upon receiving UMs, nodes in RT_LOSS state are the candidates of potential repaired route. The destination or intermediate node in RT_OK state will send Route Reply (RRPY) to confirm the reconstructed route. After the confirmation of repaired route, the CF process ends.

The process of $\mathrm{CF}$ is illustrated in Fig.1. Initially the route from source to destination is broken. Accordingly, node 2 marks the route as RT LOSS. When an UM reaches node 2, the UM is broadcasted through a subnet which consists of nodes 3 to 7 . When the UM reaches node 8, which is one of the original nodes

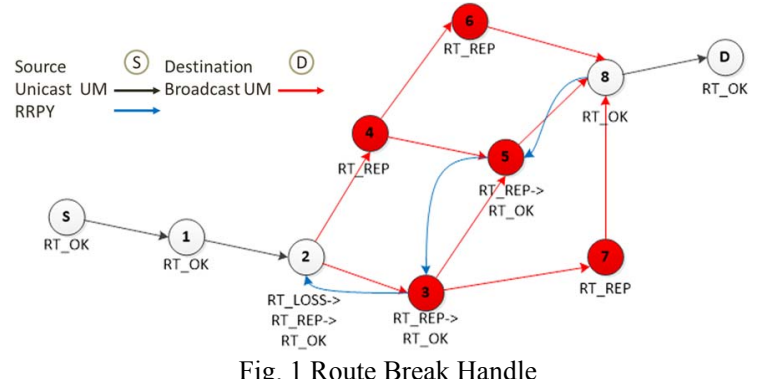

Fig. 1 Route Break Handle on the route, node 8 will forward the UM and send a RRPY back to node 5 and confirm the route. Then, UMs will be forwarded via repaired route.

The route states of the nodes locating on the remaining parts of the broken route, consisting of nodes 1, 2 and 8 , can indicate the rough direction where the destination is. With the help of remaining parts, the broadcast storm is reduced into node 2 to 8 range instead of node $\mathrm{S}$ to node $\mathrm{D}$ range. Therefore, the number of broadcast messages is dropped. Meanwhile, the end-to-end delay of broadcasting the UM is decreased as compared to the delay of transmission after reparation of the route.

\section{Passive Anti-Flooding (PAF)}

In CFPAF, Passive Anti-Flooding (PAF) is designed to terminate the flooding storm and prevent miss-operation due to late arrival UM. The PAF mechanism consists of two parts: overhearing RRPY messages and anti-flooding by REXT messages.

In CFPAF, RRPY is unicast to the next hop towards the source node. Nodes who are not the RRPY target can still hear it due to the wireless nature. PAF function is triggered by this "overhearing" feature.

If overhearing RRPY or receiving REXT happens during back-off time $r t$, nodes will stop their back-off timer, discard UMs, and enter the PAF_S state. When a node in PAF_S receives a broadcast $\mathrm{UM}$, and if it is the first time receiving this UM, the UM will be broadcasted and the node will enter the RT_LOSS state. Otherwise, the UM will be dropped. Additionally, the node will broadcast a REXT message with a $\mathrm{TTL}=2$. If a node is in either PAF_S or RT_OK state when receiving the REXT message, it will not broadcast REXT again.

The process of PAF is illustrated in Fig. 2 and Fig. 3. As shown in Fig. 2, upon receiving an UM, node 8 unicasts it to the destination. Meanwhile, node 8 sends an RRPY message back to node 5. The RRPY is overheard by neighbouring nodes, who will change their states to PAF_S.

As shown in Fig. 3, node 6 overhears the RRPY message from node 8 and subsequently receives a broadcasted UM from node 14. Node 6 broadcasts a REXT back to node 14 and node 14 re-broadcasts REXT to all its neighbours. Because of the back-off time, nodes 9,11 and 12 receive the REXT before they

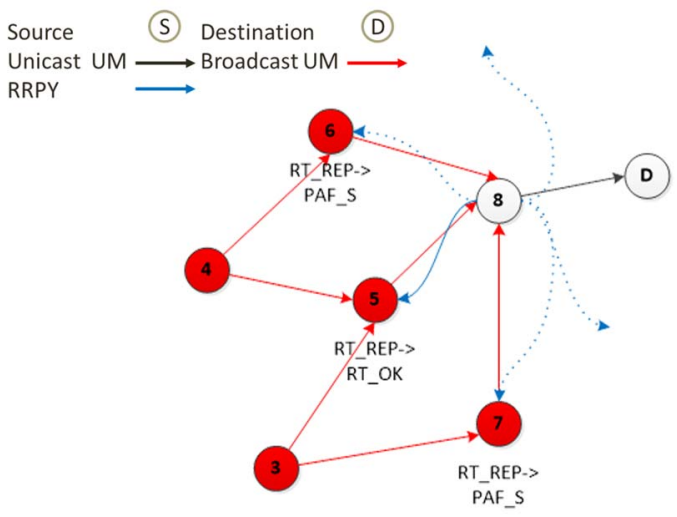

Fig. 2 Enter Overhear State 


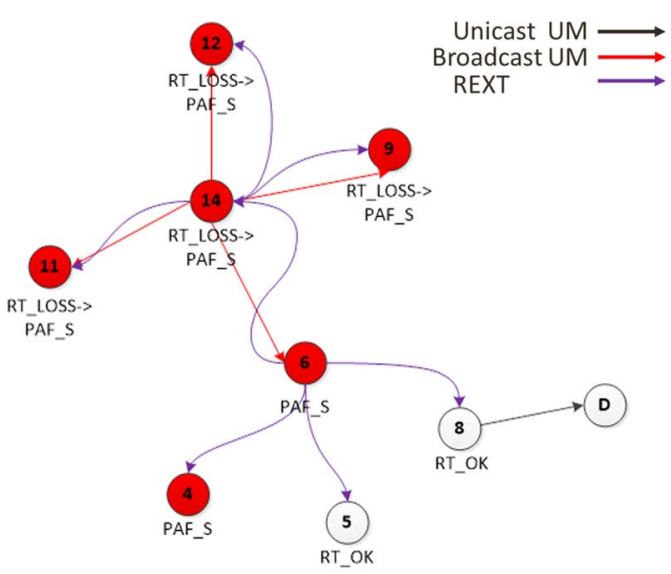

Fig. 3 Passive Anti-Flooding

broadcast the UM. So they will discard the UM. Moreover, nodes 9, 11, 12 and 14 all enter the PAF_S state. They will stay in this state until timer expire and perform PAF if they receive late arrival UMs.

Since PAF is a passive process and REXT is a small size packet and is only forwarded for two hops, PAF will not incur in excessive energy and bandwidth waste.

\section{E. Advanced Gossip Machanism (AGM)}

In order to reduce the number of UMs that are broadcasted, AGM is introduced to further reduce the number of packets of flooding. The probability $p$ of re-broadcast UM is calculated as

$$
p=\frac{\text { Neighbour_Num_UM_Num }}{\text { Neighbour_Num }},
$$

where Neighbour_Num is the number of neighbours of a given node and UM_Num is the number of UMs received during the back-off time $r t$. Therefore, the more neighbours broadcast an UM, the lower the probability that the node broadcasts the message.

As shown in Fig. 4, node 1 has 5 neighbours, thus the Neighbour_Num is 5. When node 1 receives the first message from node 2, it waits for $r t$ period. During this time, node 1 receives a message from nodes 3 and 6 . Node 1 calculates the probability $p$, which in this example is 0.4 , and uses it to decide whether to broadcast the message again. Nodes whose neighbours have received UM are less likely to broadcast again. This reduces the cost of broadcast.

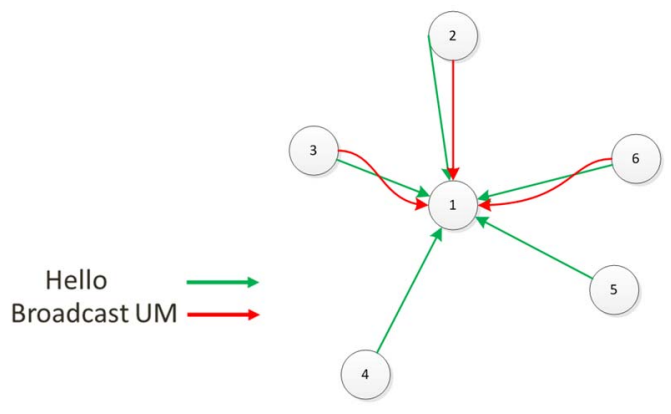

Fig. 4 Gossip

\section{SimUlation DESIGN}

\section{A. Simulation environment}

The OPNET software is adopted as a simulation platform for this study. Every scenario is simulated five times with different random seeds. If not mentioned specially, the UM generation rate is 0.5 packet per second and the simulation lasts for 5 minutes.

\section{B. Variables and fomulas for measure the performance}

The main variables and formulas used to measure the performance of the protocols are presented in table II.

\begin{tabular}{|c|c|}
\hline \multicolumn{2}{|c|}{ TABLE II VARIABLES AND FORMULAS } \\
\hline$n$ & The total number of UM sent by source. \\
\hline$H_{o p}$ num $_{i}$ & $\begin{array}{l}\text { The hop number for forwarding the } i \text { th } \mathrm{UM} \\
\text { packet. }\end{array}$ \\
\hline Total_Pkt_Num & $\begin{array}{l}\text { The sum of UM packets receives and processes } \\
\text { by each node. It is obtained by simulation. }\end{array}$ \\
\hline Unicast_Pkt_Num $_{-}$ & $\begin{array}{l}\text { The sum of unicast UM packet number. It is } \\
\text { obtained by } \sum_{i}^{n} \mathrm{Hop}_{-} \mathrm{Num} \text {. }\end{array}$ \\
\hline 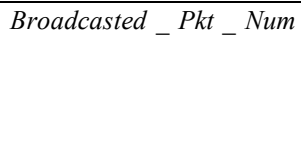 & $\begin{array}{l}\text { The sum of broadcasted UM receives and } \\
\text { processes by each node. This value is obtained } \\
\text { by } \\
\text { Broadcast_Pkt_Num-Unicast_Pkt_Num }\end{array}$ \\
\hline ir & $\begin{array}{l}\text { The packet increasing ratio. It is produced by } \\
\frac{\text { Broadcast_Pkt_Num }}{\text { Unicast_Pkt_Num }} \text {. }\end{array}$ \\
\hline
\end{tabular}

\section{Scenario one}

The first scenario is to demonstrate the relationship between performance of CFPAF and back_off value. The topology and simulation results are shown in Fig. 5. Node 1 is source and node 3 is destination. There are 30 nodes in the area. The number of UM sent by the source, $n$, is same for every sub-scenario while the average packet generation rate is 2,1 and 0.5 respectively. Thus the simulation time is different for every sub-scenario. Only CFPAF is simulated.

Lower back_off means that it is less likely to collect packets from neighbour during back off time, thus the probability of rebroadcasting an UM is higher. $5 \mathrm{~ms}$ is chosen for next two scenarios as a trade-off point of back off and ir depends on the simulating data. As shown in Fig. 5, although the Hop_num $i$ may vary due to random seeds, the curve shows the tendency of the ir versus back_off. ir drops when back_off becomes larger. When the back_off exceeds 5ms, ir reaches the bottom. Increasing of back_off is not going to further reduce the packet number.

As shown in Fig. 5, the average end-to-end delay has the same tendency as the maximum end-to-end delay. The maximum value can be 10 or more times as large as average value. It affects the average directly. The end-to-end delay increases when the back_off increases. Larger back_off may cause the later UM catches the former one UM and leads to increasing of the end-to-end delay. 


\section{Scenario two}

The second scenario contains three sub-scenarios. It demonstrates how the performance of MPF and CFPAF is affected by different position of the route within the network area. The first sub-scenario is the most common one. The route is in the middle of the network. When broadcasting, the packets affect both sides of the route. The second sub-scenario corresponds to a route at the edge of the network. The broadcasted packets only affect one side of the route. In the third sub-scenario, the route corresponds to the diagonal of the network. The route has the same number of hops as before, but has fewer neighbours around source and destination. Source and destination are shown in the Fig. 6. There are 30 nodes in the area.

Because of the multi-path strategy, the packet number of MPF is considerately high. The complex mesh style routes mean that an UM is forwarded several times by one node. But at the same time, the maximum end-to-end delay is near the average delay. MPF has better robustness compared to CFPAF. However, unlike MPF, CFPAF saves more resources by dramatically reducing the number of forwarded packets. The average end-to-end delay of CFPAF is close to MPF, whereas the maximum end-to-end delay is higher than MPF.

\section{E. Scenario three}

Scenario three demonstrates the performance of several nodes leaving the route at the same time. In BASN, nodes may move with limbs. When a nodes is moving, the route breaks into several parts and the moving nodes may send packets in parallel. Protocols need to be robust enough to handle such complicated scenario. The topology and simulation results are shown in Fig. 7. Node 1 is source and node 3 is destination. 32 nodes are in the network.

The packet number drop in MPF is due to routes reduction causing by nodes leaving the area. The average end-to-end delay
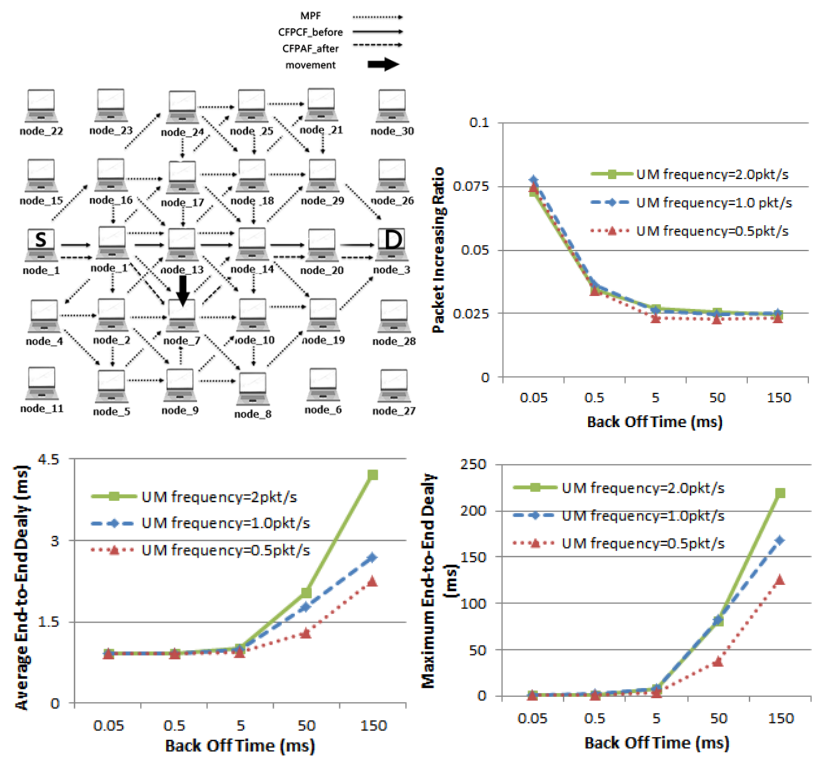

Fig. 5 Route Selection of Scenario One
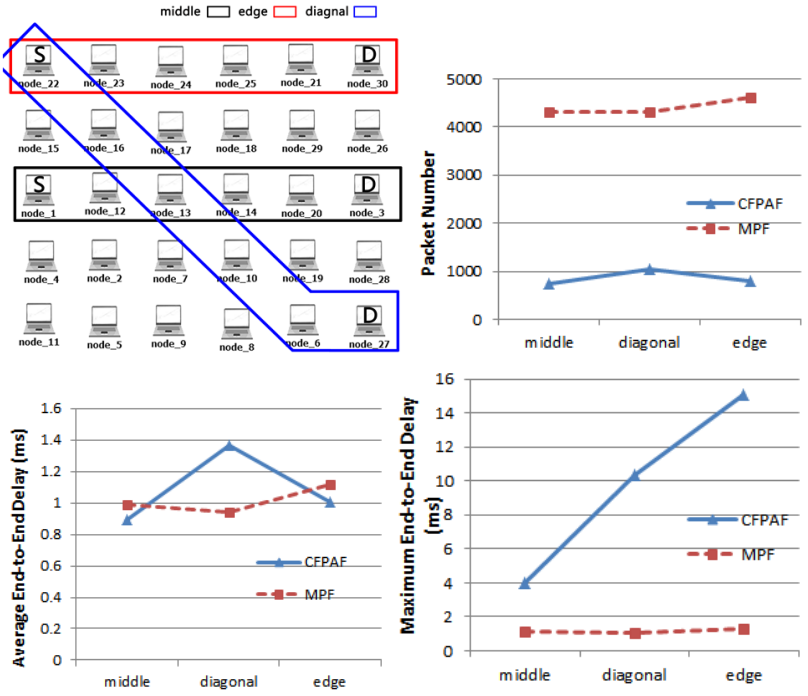

Fig. 6 Topology of Scenario Two

of MPF is almost the same with regard to the number of moving nodes. Even when there are three nodes leaving the area, the maximum end-to-end delay is close to other cases. CFPAF also performs well. Compared to 1 node leaving scenario, more packets are broadcasted in the 3-node leaving scenario. This is because of the large gap between the two remaining routes. However, the packet number of CFPAF is less than MPF. The average end-to-end delay of CFPAF is close to the same level as MPF. The increased maximum end-to-end delay is a trade-off of packet number.
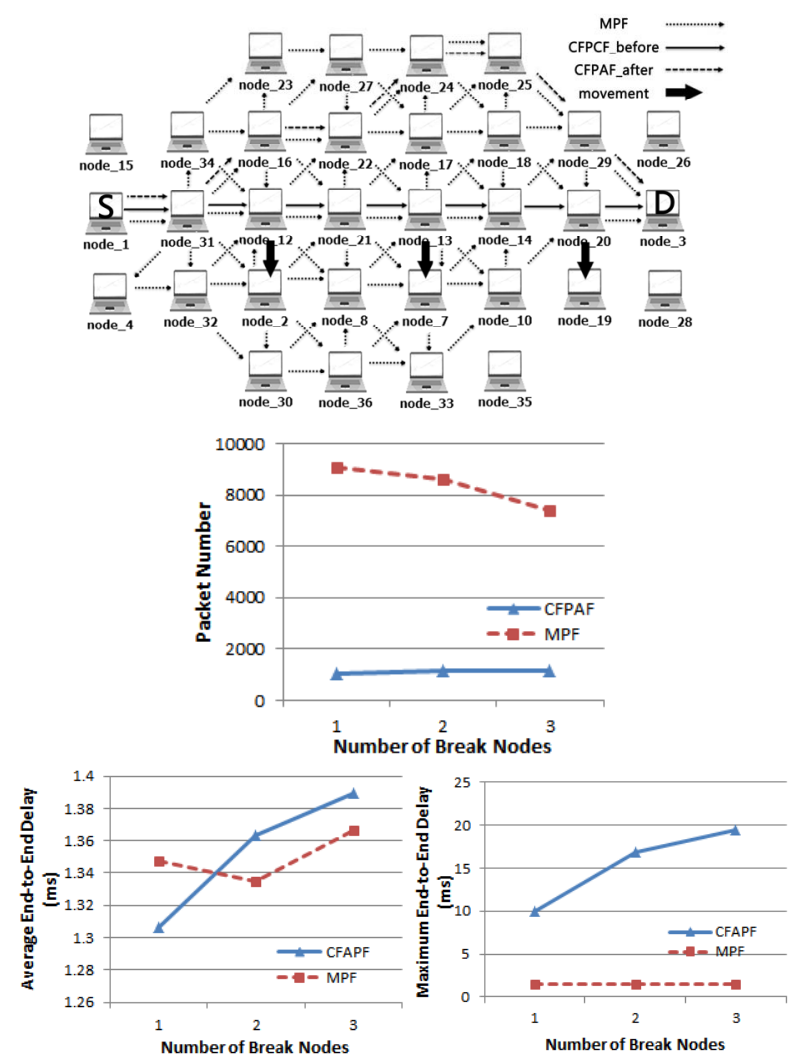

Fig. 7 Route Selection of Scenario Three 


\section{CONCLUSION}

In this paper, we have presented a new method, CFPAF, for handling UM in BASN. CFPAF aims to achieve a low end-toend delay and reduce the cost comparing to maintaining routes or flooding in whole network. The simulation results support the idea of design. Compared to conventional MPF, end-to-end delay increases moderately when dealing with route break, while the cost of flooding decreases considerably. We have also shown that when topology and total packet number are fixed, the performance is affected by the back off time. Applying this protocol in a BASN could help saving energy and make the network work longer. A modified CFPAF version can work more effectively for non-delay tolerant applications. This can be addressed in future work.

\section{REFERENCES}

[1] F. D'Andreagiovanni and A. Nardin, "Towards the fast and robust optimal design of Wireless Body Area Networks," Appl. Soft Comput., 2015.

[2] J. Zhou, A. Guo, H. T. Nguyen, and S. Su, "Intelligent Management of Multiple Access Schemes in Wireless Body Area Network," J. Networks, vol. 10, no. 2, pp. 108-116, 2015.

[3] C. A. Rypinski, "IEEE P802. 15 Wireless Personal Area Networks."

[4] L. Ciminiera, C. Demartini, and A. Valenzano, "Industrial IEEE 802.3 networks with short delivery time for urgent messages," IEEE Trans. Ind. Electron., vol. 35, no. 1, pp. 18-25, 1988.

[5] W. Kim, H. Joo, K. An, I. Lee, and H. Song, "Urgency-based packet scheduling and routing algorithms for delay-sensitive data over MANETs," Wirel. Networks, vol. 19, no. 7, pp. 1595-1609, 2013.

[6] G. Pradeep and E. Ngai, "An Adaptive Cross-Layer Design for Prioritized Traffic in Wireless Communications," Mob. Ad-hoc Sens. Networks ..., pp. 203-206, 2010.

[7] S.-H. Lee, D.-W. Kum, K.-W. Lee, and Y.-Z. Cho, "Energy-Efficient Data-Aware Routing Protocol for Wireless Sensor Networks," Int. Conf. Mob. Ubiquitous Comput. Syst. Serv. Technol., pp. 101-106, 2007.

[8] J. Chiu, C. Zheng, Y. Huang, and K. Yang, "Design and Implementation of Sequential Repair and Backup Routing Protocol for Wireless Mesh Network," pp. 1066-1070, 2014.

[9] Y.-C. Tseng, S.-Y. Ni, Y.-S. Chen, and J.-P. Sheu, "The broadcast storm problem in a mobile ad hoc network," Wirel. networks, vol. 8, no. 2-3, pp. 153-167, 2002.

[10] S. Y. Ni, Y. C. Tseng, Y. S. Chen, and J. P. Sheu, "The Broadcast Storm Problem in a Mobile Ad Hoc Network," Proc. fifth Annu. IEEE Int. Conf. Mob. Comput. Netw., pp. 151-162, 1999.

[11] Z. J. Haas and M. R. Pearlman, "The performance of query control schemes for the zone routing protocol," IEEE/ACM Trans. Netw., vol. 9, no. 4, pp. 427-438, 2001.

[12] Y.-P. Tsai, M.-C. Chung, and R.-S. Liu, "A forwarding mechanism for processing two categories of packets in wireless sensor networks," in Computer Communication Control and Automation (3CA), 2010 International Symposium on, vol. 1, pp. 466-469, 2010.

[13] M. D. D. E. Amorim and S. Fdida, "Reducing Latency and Overhead of Route Repair with Controlled Flooding," Wirel. Networks, pp. 347-358, 2004.

[14] K. Ishibashi and M. Yano, “A Proposal of Forwarding Method for Urgent Messages on an Ubiquitous Wireless Sensor Network," 6th Asia-Pacific Symp. Inf. Telecommun. Technol., pp. 293-298, 2005.

[15] R. S. H. Istepanian, E. Jovanov, and Y. T. Zhang, "Introduction to the special section on m-Health: Beyond seamless mobility and global wireless health-care connectivity," IEEE Trans. Inf. Technol. Biomed., vol. 8, no. 4, pp. 405-414, 2004.

[16] A. Busson, A. Lambert, D. Gruyer, and D. Gingras, "Analysis of Intervehicle Communication to Reduce Road Crashes," IEEE Trans. Veh. Technol., vol. 60, no. 9, pp. 4487-4496, 2011.

[17] Apple Inc. (2015, May, 17th). Now everybody can do their part to advance medical research [Online]. Available: https://www.apple.com/researchkit/ 